Research Article

\title{
Application of FWA-Artificial Fish Swarm Algorithm in the Location of Low-Carbon Cold Chain Logistics Distribution Center in Beijing-Tianjin-Hebei Metropolitan Area
}

\author{
Liyi Zhang $\mathbb{D}^{1},{ }^{1}$ Mingyue Fu $\mathbb{D}^{2},{ }^{2}$ Teng Fei $\mathbb{D},{ }^{1}$ and Xuhua Pan $\mathbb{D}^{1}$ \\ ${ }^{1}$ School of Information Engineering, Tianjin University of Commerce, Tianjin 300134, China \\ ${ }^{2}$ School of Economics, Tianjin University of Commerce, Tianjin 300134, China \\ Correspondence should be addressed to Xuhua Pan; panxuhua@tjcu.edu.cn
}

Received 29 March 2021; Accepted 7 July 2021; Published 2 August 2021

Academic Editor: Xiaobo Qu

Copyright (c) 2021 Liyi Zhang et al. This is an open access article distributed under the Creative Commons Attribution License, which permits unrestricted use, distribution, and reproduction in any medium, provided the original work is properly cited.

Green development is the hot spot of cold chain logistics today. Therefore, this paper converts carbon emission into carbon emission cost, comprehensively considers cargo damage, refrigeration, carbon emission, time window, and other factors, and establishes the optimization model of location of low-carbon cold chain logistics in the Beijing-Tianjin-Hebei metropolitan area. Aiming at the problems of the fish swarm algorithm, this paper makes full use of the fireworks algorithm and proposes an improved fish swarm algorithm on the basis of the fireworks algorithm. By introducing the explosion, Gaussian mutation, mapping and selection operations of the fireworks algorithm, the local search ability and diversity of artificial fish are enhanced. Finally, the modified algorithm is applied to optimize the model, and the results show that the location scheme of low-carbon cold chain logistics in Beijing-Tianjin-Hebei metropolitan area with the lowest total cost can be obtained by using fireworks-artificial fish swarm algorithm.

\section{Introduction}

With the rapid development of economy and the increase of people's disposable income, the demand for cold chain food such as vegetables, fruits, and dairy products is constantly increasing, and people have higher and higher requirements for the quality of cold chain food. With fresh electricity as the symbol, the cold chain industry develops rapidly, creating a huge space for the development of cold chain products. In 2020, the outbreak of COVID-19 and the closed management will further promote the development of China's online fresh e-commerce market, which is both an opportunity and a challenge for the cold chain logistics industry. On the one hand, the growth of online consumption of fresh products will promote the growth of cold chain transportation in the logistics industry and increase investment, so the cold chain logistics industry is expected to accelerate its development. On the other hand, the outbreak has also exposed problems in cold chain logistics. While the epidemic forces the fresh food industry chain to carry out a big reform, it also shows the importance of strengthening the construction of the cold chain logistics system.

In the 1980s, the United States, Japan, Western Europe, and other developed countries have basically completed the construction of the cold chain, and their food cold chain logistics is also basically perfect. However, the development of China's cold chain market lags behind that of developed countries, and there are some problems in cold chain logistics, such as low information level, insufficient infrastructure construction, and high cost. Zhou Yuan, an academician at the Chinese Academy of Sciences, pointed out that China's cold chain circulation rate is only 19 percent, while almost all foreign countries are above 85 percent [1]. The level of cold chain logistics in China is low. It is estimated that the annual loss of meat, vegetables, fruits, and aquatic products in China reaches 1.2121 million tons, 253.629 million tons, 21.957 million tons, and 8.244 tons, respectively [2].

Compared with normal temperature logistics, cold chain logistics is characterized by high carbon emission due to its 
higher requirements on transportation conditions. The CPC Central Committee with General Secretary Xi Jinping as the core has put forward the concepts of "green development" and "low-carbon economy" and emphasized the construction of ecological civilization for many times, which requires the cold chain logistics industry to reduce energy consumption and carbon emissions and promote green and low-carbon development.

Reasonable location of cold chain logistics distribution center is not only conducive to improving distribution efficiency and sales volume of fresh products, thereby reducing costs, improving the profitability of enterprises, and improving the operation efficiency and economic benefits of the whole social logistics system, but also improves service level and customer satisfaction and enhances the social influence of enterprises. This paper establishes a location model of cold chain logistics distribution center in the Beijing-Tianjin-Hebei metropolitan area with the goal of minimizing the total cost, including manufacturers, distribution centers, and customers.

The rest of this paper is as follows. In Section 2, a literature review is presented. In Section 3, a location model of low-carbon cold chain logistics distribution center is established. In Section 4, the reformative artificial fish swarm algorithm, FWA-artificial fish swarm algorithm, is elaborated in detail. In Section 5, taking the Beijing-Tianjin-Hebei metropolitan area as an example, the basic fish swarm algorithm, FWA-artificial fish swarm algorithm, and DNAartificial fish swarm algorithm are used to solve the model, and the results are analyzed. In Section 6, the conclusion is made.

\section{Literature Review}

Cold chain logistics distribution center connects manufacturers and customers, which affects the distribution efficiency of the whole logistics system. Moreover, as the most important infrastructure in cold chain logistics, distribution center has high construction cost and long recovery cycle. Once built, it will be used for a long time and difficult to migrate. Therefore, the location of distribution center has been concerned and studied by many scholars at home and abroad. Marinakis [3] integrated topological structure into the particle swarm optimization algorithm to solve the discrete optimization problem about location path. Li et al. [4] proposed a low-carbon path problem with the goal of minimizing energy consumption, carbon emissions, and total cost of car rental and proposed a tabu search algorithm based on path division to solve it. Sadjadi et al. [5] set the demand and lead time that are subject to Poisson distribution and exponential distribution, respectively, to make the problem more in line with the actual situation. On the basis of queuing analysis, a mixed integer nonlinear programming model is established to study the location problem of three-stage supply chain. Ma et al. [6] considered the change of vehicle load during driving and its influence on fuel consumption and carbon emissions and took the regional delivery network of Zhuhai Express Company as an example to analyze the low-carbon VRP model for multivehicle in a small urban area. Since the change of altitude in mountainous areas could affect the change of temperature and relative humidity and then affect the quality of perishable food, Orjuela-Castro et al. [7] took the capital of Colombia as an example and proposed a site selection model for the transportation of perishable food in mountainous areas. El-Sobky et al. [8] used the trust region method to improve the ability of global convergence and combined the penalty method and active set strategy to determine the number and scale of warehouses with the goal of minimal cost. Simic et al. [9] analyzed the problem of facility location, proposed the methodology based on the fuzzy C-means method, analytic hierarchy process, and hybrid genetic algorithm, and explained how to analyze the multicriteria decision making problem with this model. Wang et al. [10] used the improved genetic and particle swarm algorithm and the minimum and maximum costbenefit distribution model to solve the benefit distribution problem of multicenter joint distribution. Kang et al. [11] established a research on the optimization of low-carbon distribution path of cold chain logistics of fresh agricultural products and analyzed it with the improved ant colony algorithm. Liang et al. [12], respectively, built the path models of traditional gasoline vehicles and electric vehicles in a low-carbon environment to study the impact of electric vehicles on economic cost and carbon emissions. Bilisik et al. [13] used interval valued trapezoidal fuzzy grey relational degree analysis to solve the distribution location problem of fresh food in urban areas. Zhang et al. [14] used the inputoutput method to calculate the carbon emission cost, and combined with customers' time satisfaction and quality satisfaction, they compared and analyzed the difference of distribution cost between the single model and multimodel. Sheng [15] proposed the quantum particle swarm optimization algorithm and used it to optimize the logistics management system. Park et al. [16] analyzed the factors influencing the intelligent distribution center, which provided a certain theoretical basis for the construction of intelligent distribution center. Setiawan et al. [17] established three resource allocation models to enable the selected distribution center to allocate rescue resources effectively and quickly in the face of sudden disasters. Zhou et al. [18] proposed a new urban distribution model based on subway by studying the urban subway network and road network. Song et al. [19] established an emergency logistics system by comprehensively considering the delivery time and cost under the condition of material demand and dynamic road conditions. Li et al. [20], under the multichannel urban network, measured carbon emissions by vehicle speed and load and constructed a low-carbon path optimization model. Meng and Gao [21] improved the moth optimization algorithm by using the nonlinear inertia weight based on control factors and the cross-mutation strategy in the genetic algorithm and optimized the location model of agricultural logistics distribution center with the modified moth optimization algorithm. Li and Dang [22] used the heuristic algorithm to solve the logistics site-optimization model under constraints such as vehicle capacity, cargo operation time, and delivery operation time window in the case of 
multiple distribution centers. Li et al. [23] studied the distribution problem of dairy products in Beijing by constructing a two-level distribution site-path model with constraints. Lin et al. [24] adopted stochastic programming theory to study the location of low-carbon logistics distribution center in the case of uncertain demand. Xu et al. [25] proposed a pseudo-reverse spider monkey optimization algorithm based on Laplace distribution to solve the location problem of logistics distribution centers.

From the above literatures, most of them study location models that only include distribution centers and demand points, while few study three-level models that include manufacturers, distribution centers, and customers and also contain fewer types of costs. Therefore, this paper first establishes a three-level model including constant cost, transportation cost, cargo damage cost, cooling cost, carbon emission cost, and penalty cost. Secondly, the fireworks algorithm is integrated into the fish swarm algorithm, and FWA-artificial fish swarm algorithm is proposed and used to solve the model.

\section{Model}

The total cost of this model includes constant cost, transportation cost, cargo damage cost, cooling cost, carbon emission cost, and penalty cost. The parameters of the model are as follows:

$I$ : the number of manufacturers, $i=1,2, \ldots, I$

$J$ : the number of distribution centers, $j=1,2, \ldots, J$

$M$ : the number of customers, $m=1,2, \ldots, M$

$\alpha_{j}$ : when the distribution center is selected, it is 1 ; otherwise, it is 0 .

$\alpha_{j m:}$ when customer is delivered by distribution center, it is 1 ; otherwise, it is 0 .

$c_{j}$ : the cost of distribution center $j$ remains unchanged in the short term $p_{2}$ : unit transportation cost

$d_{i j}$ : distance between supplier $i$ and distribution center $j$

$d_{j m}$ : distance between distribution center $j$ and customer $m$

$q_{i j}$ : demand of distribution center $j$

$q_{j m}$ : demand of customer $m$

$p_{3}$ : food's unit price

$T_{m}$ : the time required to uninstall food

$v$ : speed of vehicle

$\beta_{1}$ : percentage of food spoilage in transit

$\beta_{2}$ : percentage of food spoilage at unloading stage

$p_{41}$ : the cost of keeping the temperature low in transit

$p_{42}$ : the cost of keeping the temperature low at unloading stage

$E_{1}$ : fuel consumption of vehicle driving $1 \mathrm{~km}$

$E_{2}$ : the energy consumed per hour due to cooling

$p_{c}$ : the amount to be paid for generating one unit of carbon dioxide due to energy consumption

$e$ : the amount of carbon dioxide produced per unit of energy consumed

$p_{61}$ : cost of a vehicle arriving one hour earlier

$p_{62}$ : the cost incurred when a vehicle is one hour late $t_{m}$ : actual delivery time to customer $m$

$\left(E T_{m}, L T_{m}\right)$ : the best time for vehicles to reach customer $m$

$\left(E E T_{m}, L L T_{m}\right)$ : the maximum time period for vehicles to arrive. If this time period gets exceeded, food will not be accepted by customers

The location model of low-carbon cold chain logistics distribution center is as follows:

$$
\begin{aligned}
\min C_{t} & =C_{1}+C_{2}+C_{3}+C_{4}+C_{5}+C_{6}, \\
C_{1} & =\sum_{j=1}^{J} c_{j} \alpha_{j}, \\
C_{2} & =p_{2} \sum_{i=1}^{I} \sum_{j=1}^{J} \sum_{m=1}^{M}\left(q_{i j} d_{i j} \alpha_{j}+q_{j m} d_{j m} \alpha_{j m}\right), \\
C_{3} & =p_{3} \sum_{i=1}^{I} \sum_{j=1}^{J} \sum_{m=1}^{M}\left(q_{i j} \alpha_{j}\left(1-e^{-\beta_{1} t_{i j}}\right)+q_{j m} \alpha_{j m}\left(1-e^{-\beta_{1} t_{j m}}\right)+q_{j m} \alpha_{j m}\left(1-e^{-\beta_{2} T_{m}}\right)\right), \\
C_{4} & =\sum_{i=1}^{I} \sum_{j=1}^{J} \sum_{m=1}^{M}\left(p_{41}\left(t_{i j} \alpha_{j}+t_{j m} \alpha_{j m}\right)+p_{42} T_{m} \alpha_{j m}\right),
\end{aligned}
$$




$$
\begin{aligned}
C_{5} & =p_{c} e E_{1} \sum_{i=1}^{I} \sum_{j=1}^{J} \sum_{m=1}^{M}\left(d_{i j} \alpha_{j}+d_{j m} \alpha_{j m}\right)+p_{c} e E_{2} \sum_{i=1}^{I} \sum_{j=1}^{J} \sum_{m=1}^{M}\left(t_{i j} \alpha_{j}+t_{j m} \alpha_{j m}\right), \\
C_{6}(m) & = \begin{cases}\text { inf, } & t_{m}<E E T_{m}, \\
p_{61}\left(E T_{m}-t_{m}\right), & E E T_{m}<t_{m}<E T_{m}, \\
0, & E T_{m}<t_{m}<L T_{m}, \\
p_{62}\left(t_{m}-L T_{m}\right) & L T_{m}<t_{m}<L L T_{m}, \\
\inf , & t_{m}>L L T_{m},\end{cases} \\
C_{6} & =\sum_{m=1}^{M} C_{6}(m) .
\end{aligned}
$$

Constraints are as follows:

$$
\begin{gathered}
\sum_{j=1}^{J} \alpha_{j m}=1, \quad m \in M, \\
\sum_{j=1}^{J} \alpha_{j} \leq N \\
\sum_{i=1}^{I} \sum_{j=1}^{J} q_{i j}=\sum_{j=1}^{J} \sum_{m=1}^{M} q_{j m}, \\
\sum_{n=1}^{N} V_{n} \geq \sum_{m=1}^{M} q_{m} .
\end{gathered}
$$

Formula (1) is an objective function, which aims at minimizing the total cost $C_{t}$. In formula (2), $C_{1}$ represents the total constant cost in the system; in equation (3), $C_{2}$ represents the total transportation cost from the manufacturer to distribution centers and from distribution centers to the customers; in formula (4), the total cost of cargo damage $C_{3}$ refers to the sum of the cost of spoilage in transit and the cost of spoilage when unloading at customers; in equation (5), the total cooling cost $C_{4}$ refers to the total cooling cost during transportation and unloading. In formula (6), the total carbon emission cost $C_{5}$ is the cost of carbon dioxide caused by the consumption of fuel by vehicles and energy consumption by refrigeration equipment. Equation (7) represents the penalty cost caused by the vehicle arriving at the customer $m$ late or early; in formula (8), $C_{6}$ is the total penalty cost. Formula (9) indicates that a customer's demand can only be delivered once. Formula (10) specifies distribution centers' maximum number $N$. Equation (11) means that the total demand of distribution centers and customers is equal. Formula (12) indicates that the total capacity of the selected distribution centers is not less than the total demand for customers.

\section{FWA-Artificial Fish Swarm Algorithm}

4.1. Artificial Fish Swarm Algorithm's Behavior Description. Let rand be a number that is randomly selected from the digital range $(0,1), \delta$ be crowding factor, and $X=\left(x_{1}, x_{2}, \ldots, x_{n}\right)$ be every artificial fish's present state. $Y=\mathrm{f}(X)$ represents the amount of food that exist in the fish's present state, where $x_{i}(i=1,2, \ldots, n)$ is defined as the desired optimization variate and $Y$ represents objective function value.

(1) Prey Behavior. At time $t$, the artificial fish's location is $x_{i}$, and another uncertainly chose position within the visual field is set as $x_{j}$. In maximum value problem, if $Y_{i}<Y_{j}$ holds, then go forward in the orientation of $x_{j}$; otherwise, choose again randomly and identify if the advancing criterion is met. If advancing criterion is still not met when the maximum number of attempts is reached, it can be randomly moved one step.

$$
\begin{cases}x_{i}^{t+1}=x_{i}^{t}+\operatorname{rand} \times \operatorname{step} \times \frac{x_{j}-x_{i}^{t}}{\left\|x_{j}-x_{i}^{t}\right\|}, & Y_{i}<Y_{j}, \\ x_{i}^{t+1}=x_{i}^{t}+\text { rand } \times \text { step }, & \text { else. }\end{cases}
$$

(2) Swarm Behavior. At time $t, x_{i}$ is the artificial fish's present location and $Y_{i}$ is set as corresponding food concentration. At present, the number of artificial fish in vision field is $n_{f}$, and central position is $x_{c}$, and $Y_{c}$ is the matching food concentration. If $Y_{c} / n_{f}>\delta Y_{i}$ is satisfied, which means that there are more artificial fish at the central position $x_{c}$ and they are uncrowded, then the fish will move toward the central location $x_{c}$ by one step. If not, the fish will perform prey behavior. 


$$
\begin{cases}x_{i}^{t+1}=x_{i}^{t}+\operatorname{rand} \times \operatorname{step} \times \frac{x_{c}-x_{i}^{t}}{\left\|x_{c}-x_{i}^{t}\right\|}, & \frac{Y_{c}}{n_{f}}>\delta Y_{i}, \\ \text { prey behavior, } & \text { else. }\end{cases}
$$

(3) Follow Behavior. At time $t, x_{i}$ is the artificial fish's current location and $Y_{i}$ is set as corresponding food concentration. In its field of vision, the food concentration $Y_{m}$ is the highest when the artificial fish state is $x_{m}$. If $Y_{m} / n_{f}>\delta Y_{i}$ is satisfied, which means that there exists a high food concentration in position $x_{m}$ and the position is uncrowded, then the fish in $x_{i}$ will move in the direction of $x_{m}$ by one step; otherwise, it will perform prey behavior.

$$
\begin{cases}x_{i}^{t+1}=x_{i}^{t}+\operatorname{rand} \times \operatorname{step} \times \frac{x_{m}-x_{i}^{t}}{\left\|x_{m}-x_{i}^{t}\right\|}, & \frac{Y_{m}}{n_{f}}>\delta Y_{i}, \\ \text { prey behavior, } & \text { else. }\end{cases}
$$

(4) Random Behavior. Random behavior is the act of randomly selecting a location in the field of vision and moving one step in that direction to expand its range of food and companions.

4.2. FWA-AFSA. The basic fish swarm algorithm has the advantages of simplicity, flexibility, and strong parallel processing capabilities. However, in the late stage of operation, the optimization speed of the algorithm is slow, and it is easy to fall into the vicinity of the local extreme value, resulting in low solution accuracy. Therefore, considering the problems of the fish swarm algorithm in the late stage, this paper introduces the fireworks algorithm into the fish swarm algorithm. The basic idea is that after multiple iterations, if the state of the optimal artificial fish does not change or the change is extremely small, the optimal artificial fish individual is retained, and explosion and Gaussian mutation operators are performed on the remaining artificial fish with a certain probability. After the explosion and Gaussian mutation operations, keep all individuals within the defined range through the mapping rule. Finally, according to the distance-based selection strategy, the better individuals are selected from the newly generated individuals to form the next-generation population with the best individuals retained from the previous generation. The explosion operator enhances the local search ability of the algorithm by enhancing the degree of information exchange in the fish school and then increases the convergence speed. The Gaussian mutation operator can generate individuals far away from the current optimal position and increase the diversity of the population.

4.2.1. Explosion Operator. Explosion operator refers to the fireworks in its vicinity to find fireworks with better fitness value. Fireworks have different explosion radius and explosion spark number. Fireworks with good fitness value can produce more sparks in a smaller explosion radius, which makes the local search ability stronger. Fireworks with poor fitness value can generate fewer sparks in a larger explosion radius, thus enabling the algorithm to have better global search capability [26].

4.2.2. Gaussian Variation. Gaussian mutation operator can generate Gaussian sparks, which can enhance the diversity of the population, thereby improving the optimization efficiency of the algorithm and avoiding falling into local extremes to a certain extent.

4.2.3. Mapping Rule. When the sparks generated by the explosion and Gaussian mutation operation exceeds the value range of the problem to be solved, the mapping rule is used to modify it.

4.2.4. Selection Strategy. After ensuring that fireworks, explosion sparks, and Gaussian mutation sparks (candidate set) are all in the search space, some of them need to be selected as the next-generation fireworks so that the excellent information in the population can be retained.

\section{Simulation}

In this paper, the Binhai New Area $(117.68,39.03)$ is selected as the manufacturer, and 13 municipal administrative units in the Beijing-Tianjin-Hebei metropolitan area are selected as the alternative distribution centers to provide service to customers. Tables 1 and 2 represent information about customers and alternative distribution centers, respectively.

Suppose the price of cold chain food is 3000 yuan/t, the transportation cost of unit food is 0.3 yuan $/ \mathrm{km}$, percentage of food spoilage in transit and at unloading stage are 0.03 and 0.05 , respectively, the unloading time is proportional to the unloading volume, and the proportional coefficient is $5 /$ 60 , the costs of vehicle arriving early and arriving late for vehicles are 10 yuan/h and 30 yuan/h, respectively, and the cooling cost in order to reduce loss during transportation and unloading is $20 \mathrm{yuan} / \mathrm{h}$ and $30 \mathrm{yuan} / \mathrm{h}$, respectively. The vehicle departs from the distribution center at $4: 30$, the driving speed is $60 \mathrm{~km} / \mathrm{h}$, the fuel consumption of the vehicle is $0.225 \mathrm{~L} / \mathrm{km}$, the energy consumption of the refrigeration equipment is $0.0025 \mathrm{~L} / \mathrm{t} . \mathrm{km}$, the carbon dioxide emission coefficient is $2.66 \mathrm{~kg} / \mathrm{L}$, and the unit carbon tax price is 20 yuan $/ \mathrm{kg}$ [27].

In this paper, the basic fish swarm algorithm (AFSA), DNA-artificial fish swarm algorithm (DNA-AFSA) [28], and FWA-artificial fish swarm algorithm (FWA-AFSA) are used to solve the location problem of the logistics center, respectively. Parameters of algorithms are set as follows. The number of artificial fish fishnum $=20$, crowding factor $\delta=0.618$, maximum number of trials try_number $=20$, step size step $=25$, viewable size visual $=80$, the probability of DNA crossing $P_{c}=0.9$, the probability of DNA mutation 
TABLE 1: Information about customers.

\begin{tabular}{|c|c|c|c|c|c|}
\hline No. & Customer & Longitude and latitude & Maximum time period & Best time period & Quantity demand \\
\hline 1 & Huairou District, Beijing & $(116.63,40.32)$ & $5: 00-8: 45$ & $5: 10-8: 15$ & 5 \\
\hline 2 & Fangshan District, Beijing & $(116.13,39.75)$ & $4: 30-9: 10$ & $4: 50-8: 30$ & 4.5 \\
\hline 3 & Jinghai District, Tianjin & $(116.92,38.93)$ & $4: 30-9: 15$ & $4: 45-9: 00$ & 4.5 \\
\hline 4 & Ninghe County, Tianjin & $(117.82,39.33)$ & $4: 30-9: 30$ & $4: 50-9: 10$ & 5 \\
\hline 5 & Pingshan County, Shijiazhuang & $(114.20,38.25)$ & $4: 45-8: 20$ & $5: 00-8: 00$ & 2.5 \\
\hline 6 & Xinji City, Shijiazhuang & $(115.22,37.92)$ & $5: 10-8: 00$ & $5: 20-7: 30$ & 4 \\
\hline 7 & Fengning County, Chengde & $(116.65,41.20)$ & $5: 45-9: 20$ & $6: 00-8: 50$ & 3 \\
\hline 8 & Longhua County, Chengde & $(117.72,41.32)$ & $4: 30-9: 30$ & $4: 40-9: 15$ & 4 \\
\hline 9 & Chongli County, Zhangjiakou & $(115.27,40.97)$ & $5: 00-9: 40$ & $5: 15-9: 00$ & 4.5 \\
\hline 10 & Huai'an County, Zhangjiakou & $(114.42,40.67)$ & $4: 45-9: 40$ & $5: 15-9: 15$ & 5 \\
\hline 11 & Changli County, Qinhuangdao & $(119.17,39.70)$ & $4: 30-9: 15$ & $4: 45-8: 45$ & 2 \\
\hline 12 & Qinglong County, Qinhuangdao & $(118.95,40.40)$ & $4: 40-9: 00$ & $5: 00-8: 30$ & 4.5 \\
\hline 13 & Gu' an County, Langfang & $(116.30,39.43)$ & $4: 30-9: 30$ & $4: 40-8: 50$ & 3 \\
\hline 14 & Xianghe County, Langfang & $(117.00,39.77)$ & $4: 45-8: 50$ & $4: 50-8: 30$ & 2.5 \\
\hline 15 & Zunhua City, Tangshan & $(117.95,40.18)$ & $5: 00-9: 15$ & $5: 10-9: 00$ & 4 \\
\hline 16 & Tanghai County, Tangshan & $(118.45,39.27)$ & $4: 30-9: 30$ & $4: 40-9: 00$ & 2 \\
\hline 17 & Laisyuan County, Baoding & $(114.68,39.35)$ & $4: 30-9: 45$ & $4: 40-9: 15$ & 5.5 \\
\hline 18 & Shunping County, Baoding & $(115.13,38.83)$ & $4: 30-8: 45$ & $4: 35-8: 15$ & 4.5 \\
\hline 19 & Renqiu City, Cangzhou & $(116.10,38.72)$ & $4: 30-9: 10$ & $4: 45-8: 30$ & 4 \\
\hline 20 & Haixing County, Cangzhou & $(117.48,38.13)$ & $5: 00-9: 40$ & $5: 15-9: 00$ & 4.5 \\
\hline 21 & Fucheng County, Hengshui & $(116.15,37.87)$ & $4: 50-8: 30$ & $5: 15-8: 00$ & 3.5 \\
\hline 22 & Gucheng County, Hengshui & $(115.97,37.35)$ & $4: 45-9: 15$ & $5: 10-9: 00$ & 5.5 \\
\hline 23 & Lincheng County, Xingtai & $(114.50,37.43)$ & $4: 30-9: 30$ & $4: 50-8: 40$ & 4.5 \\
\hline 24 & Guangzong County, Xingtai & $(115.15,37.07)$ & $4: 40-9: 15$ & $5: 15-8: 40$ & 4 \\
\hline 25 & Shexian County, Handan & $(113.67,36.57)$ & $4: 30-9: 30$ & $4: 50-8: 45$ & 4.5 \\
\hline 26 & Wei County, Handan & $(114.93,36.37)$ & $4: 40-9: 40$ & $5: 00-8: 50$ & 5 \\
\hline
\end{tabular}

TABLE 2: Information about distribution centers.

\begin{tabular}{lccc}
\hline Distribution center & Longitude and latitude & Capacity & Constant cost \\
\hline Beijing City & $(116.42,39.92)$ & 22 & 8000 \\
Tianjin City & $(117.20,39.13)$ & 26 & 27 \\
Shijiazhuang City & $(114.30,38.02)$ & 21 & 7500 \\
Chengde City & $(117.57,40.59)$ & 24 & 5000 \\
Zhangjiakou City & $(114.53,40.48)$ & 22 & 7000 \\
Qinhuangdao City & $(119.35,39.55)$ & 24 & 5000 \\
Langfang City & $(116.70,39.52)$ & 22 & 6000 \\
Tangshan City & $(118.11,39.36)$ & 27 & 5500 \\
Baoding City & $(115.30,38.51)$ & 22 & 6500 \\
Cangzhou City & $(116.52,38.18)$ & 26 & 5000 \\
Hengshui City & $(115.42,37.44)$ & 22 & 6500 \\
Xingtai City & $(114.30,37.04)$ & 24 & 5500 \\
Handan City & $(114.28,36.36)$ & 6000 \\
\hline
\end{tabular}

TABLE 3: The running results of three algorithms.

\begin{tabular}{lccccc}
\hline & Minimum value & Maximum value & Mean value & Mean convergence algebra & Standard deviation \\
\hline AFSA & 167060.58 & 235106.86 & 195877.54 & 87.87 & 16249.64 \\
DNA-AFSA & 163934.82 & 211482.97 & 186939.75 & 67.63 & 12195.75 \\
FWA-AFSA & 157539.41 & 204585.40 & 182149.97 & 54.70 & 11229.78 \\
\hline
\end{tabular}

$P_{m}=0.01$, maximum number of sparks produced by fireworks explosion $E_{n}=20$, the limiting factor for the number of explosion $a=0.3$ and $b=0.8$, explosion radius $E_{r}=500$, variation spark number $M=8$, maximum number of iterations maxgen $=100$, and threshold of the maximum number of iterations when there is no change Maxbest $=5$.
The three algorithms select 5 from 13 alternative distribution centers to deliver goods to 26 customers, and the results of running the algorithms for 30 times are shown in Table 3.

According to Table 3, in terms of solving accuracy, the minimum value, maximum value, and mean value obtained 


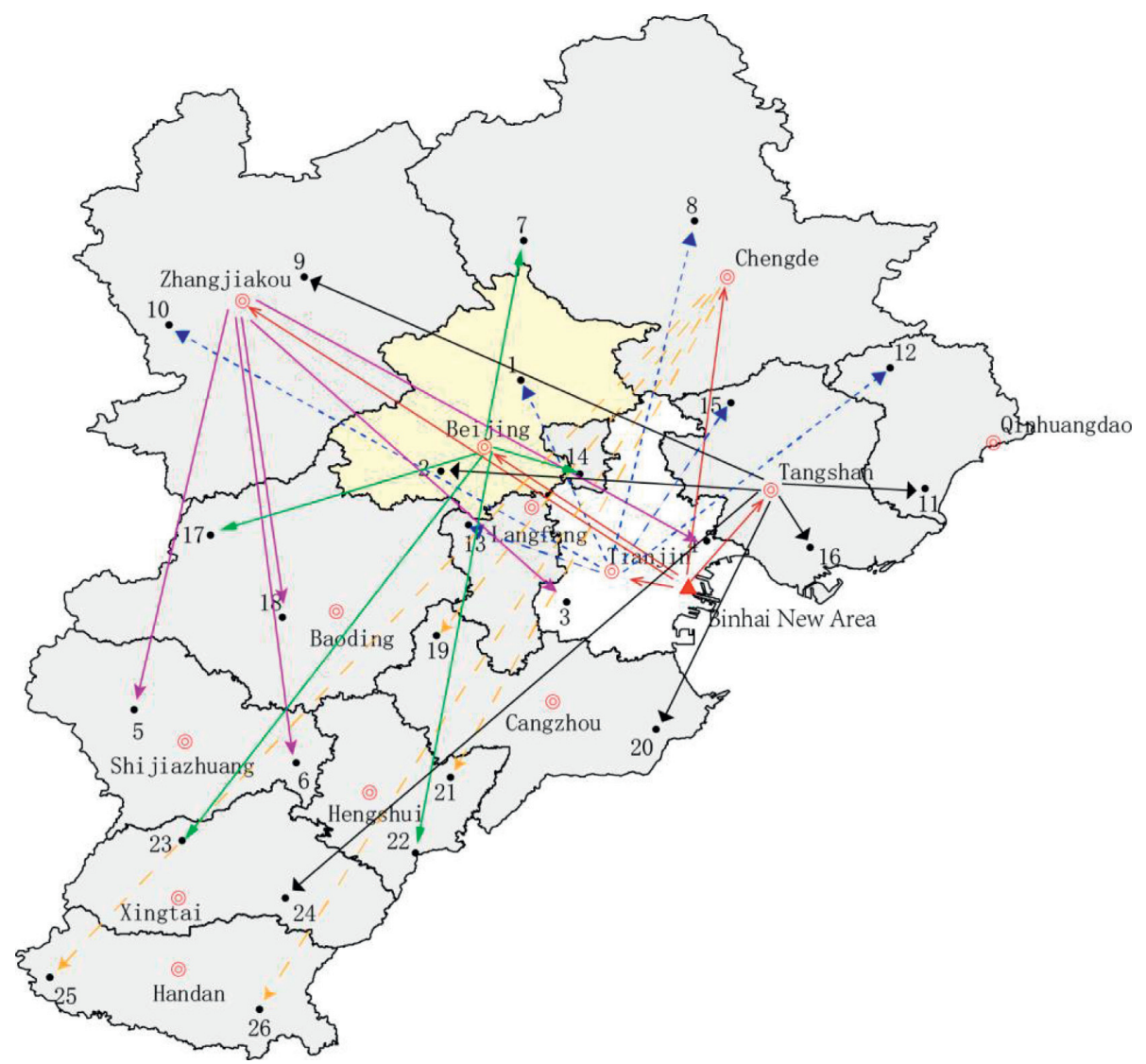

FIGURE 1: Schematic diagram of location selection of the basic artificial fish swarm algorithm.

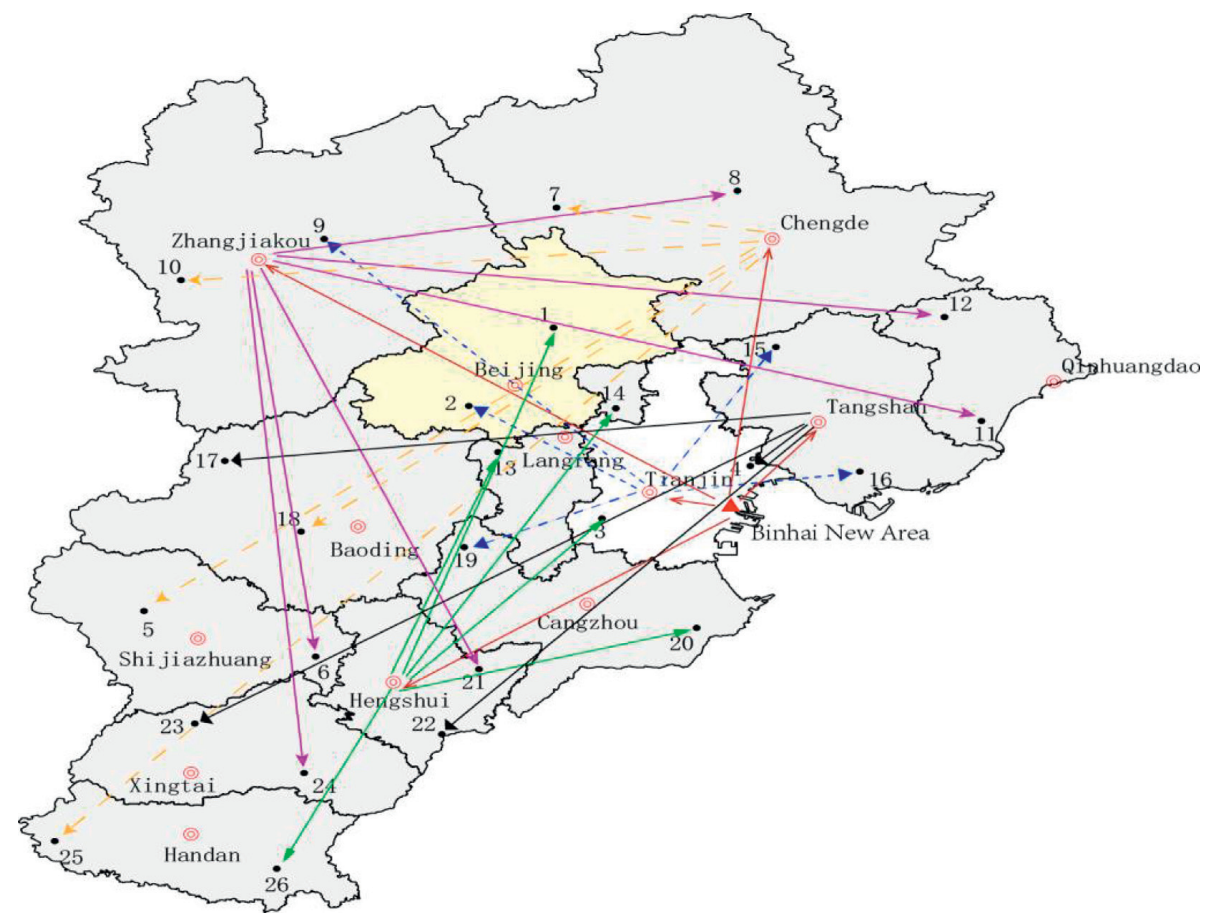

FIGURE 2: Schematic diagram of location selection of the DNA-artificial fish swarm algorithm. 


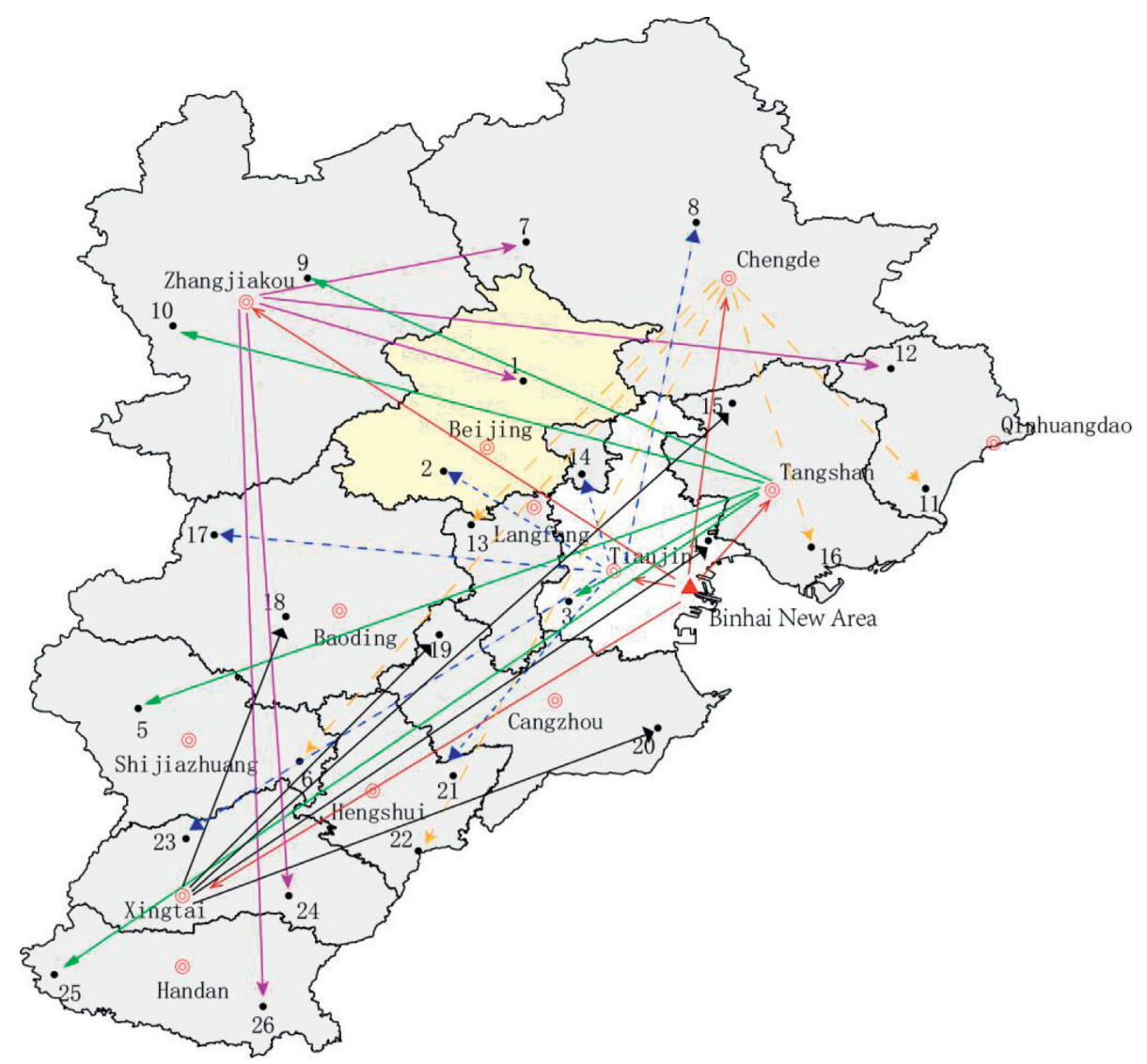

FIgURE 3: Schematic diagram of location selection of the FWA-artificial fish swarm algorithm.

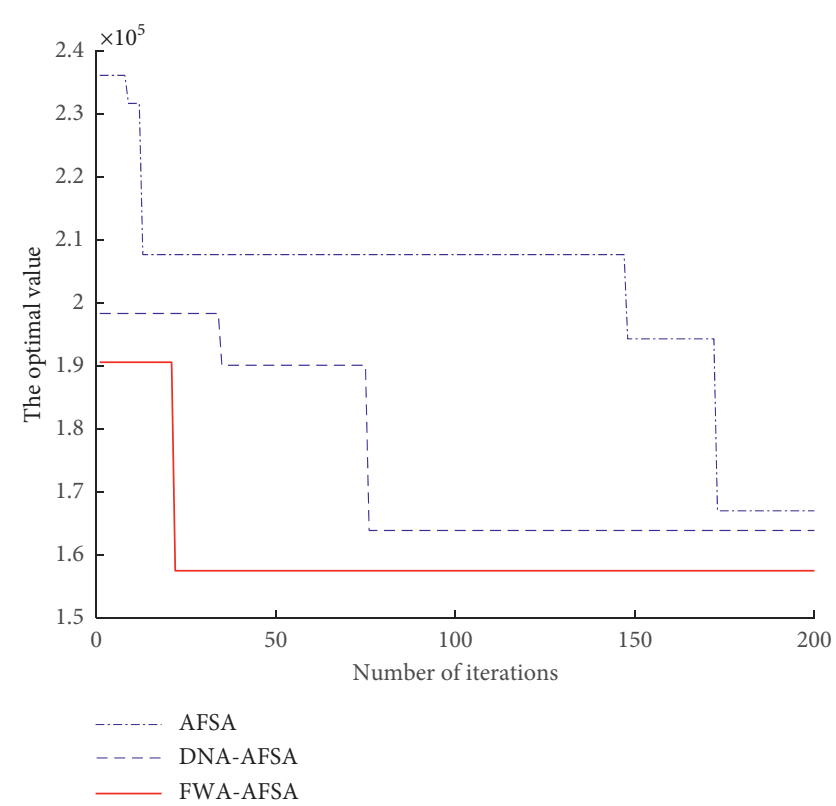

Figure 4: Comparison of optimization curves of the three algorithms.

by FWA-AFSA are, respectively, reduced by 9520.87 , 30521.46 and 13727.57 compared with those of the basic artificial fish swarm algorithm and, respectively, reduced by
6395.41, 6897.57 and 4789.78 compared with those of DNAAFSA, indicating that FWA-AFSA can obtain a lower total cost. In terms of stability, FWA-AFSA has a smaller standard deviation, which proves its stability. In terms of convergence rate, the FWA-AFSA's average convergence algebra is smallest, indicating its faster convergence rate.

The schematic diagrams of optimal location solved by the basic artificial fish swarm algorithm, DNA-artificial fish swarm algorithm, and FWA-artificial fish swarm algorithm are shown in Figures 1-3, respectively.

According to Figure 1, the optimal site selection scheme obtained by the basic artificial fish swarm algorithm is as follows: first of all, food is transported from the supplier, Binhai New Area, to 5 distribution centers: Beijing, Tianjin, Chengde, Zhangjiakou, and Tangshan. Next, the food is transported by distribution centers to customers: (1) customers transported by Beijing: 7 (Fengning County, Chengde), 14 (Xianghe County, Langfang), 17 (Laiyuan County, Baoding), 22 (Gucheng County, Hengshui), and 23 (Lincheng County, Xingtai); (2) customers transported by Tianjin: 1 (Huairou District, Beijing), 8 (Longhua County, Chengde), 10 (Huai'an County, Zhangiakou), 12 (Qinglong County, Qinhuangdao), 13 (Gu' an County, Langfang), and 15 (Zunhua City, Tangshan); (3) customers transported by Chengde: 19 (Renqiu County, Cangzhou), 21 (Fucheng County, Hengshui), 25 (Shexian County, Handan), and 26 (Wei County, Handan); (4) customers transported by Zhangjiakou: 3 (Jinghai District, Tianjin), 4 (Ninghai 
County, Tianjin), 5 (Pingshan County, Shijiazhuang), 6 (Xinji City, Shijiazhuang), and 18 (Shunping County, Baoding); (5) customers transported by Tangshan: 2 (Fangshan District, Beijing), 9 (Chongli County, Zhangjiakou), 11 (Changli County, Qinhuangdao), 16 (Tanghai County, Tangshan), 20 (Haixing County, Cangzhou), and 24 (Guangzong County, Xingtai).

According to Figure 2, the optimal site selection scheme solved by DNA-artificial fish swarm algorithm is as follows: first of all, food is transported from the supplier, Binhai New Area, to 5 distribution centers: Tianjin, Chengde, Zhangjiakou, Tangshan, and Hengshui; next, the food is transported by distribution centers to customers: (1) customers transported by Tianjin: 2 (Fangshan District, Beijing), 9 (Chongli County, Zhangjiakou), 15 (Zunhua City, Tangshan), 16 (Tanghai County, Tangshan), and 19 (Renqiu County, Cangzhou); (2) customers transported by Chengde: 5 (Pingshan County, Shijiazhuang), 7 (Fengning County, Chengde), 10 (Huai' an County, Zhangiiakou), 18 (Shunping County, Baoding), and 25 (Shexian County, Handan); (3) customers transported by Zhangjiakou: 6 (Xinji City, Shijiazhuang), 8 (Longhua County, Chengde), 11 (Changli County, Qinhuangdao), 12 (Qinglong County, Qinhuangdao), 21 (Fucheng County, Hengshui), and 24 (Guangzong County, Xingtai); (4) customers transported by Tangshan: 4 (Ninghai County, Tianjin), 17 (Laiyuan County, Baoding), 22 (Gucheng County, Hengshui), and 23 (Lincheng County, Xingtai); (5) customers transported by Hengshui: 1 (Huairou District, Beijing), 3 (Jinghai District, Tianjin), 13 (Gu' an County, Langfang), 14 (Xianghe County, Langfang), 20 (Haixing County, Cangzhou), and 26 (Wei County, Handan).

According to Figure 3, the optimal site selection scheme solved by FWA-artificial fish swarm algorithm is as follows: First, food is transported from the supplier, Binhai New Area, to 5 distribution centers: Tianjin, Chengde, Zhangjiakou, Tangshan, and Xingtai; Next, the food is transported by distribution centers to customers: (1) customers transported by Tianjin: 2 (Fangshan District, Beijing), 8 (Longhua County, Chengde), 14 (Xianghe County, Langfang), 17 (Laiyuan County, Baoding), 21 (Fucheng County, Hengshui), and 23 (Lincheng County, Xingtai); (2) customers transported by Chengde: 6 (Xinji City, Shijiazhuang), 11 (Changli County, Qinhuangdao), 13 (Gu' an County, Langfang), 16 (Tanghai County, Tangshan), and 22 (Gucheng County, Hengshui); (3) customers transported by Zhangjiakou: 1 (Huairou District, Beijing), 7 (Fengning County, Chengde), 12 (Qinglong County, Qinhuangdao), 24 (Guangzong County, Xingtai), and 26 (Wei County, Handan); (4) customers transported by Tangshan: 3 (Jinghai District, Tianjin), 5 (Pingshan County, Shijiazhuang), 9 (Chongli County, Zhangjiakou), 10 (Huai' an County, Zhangjiakou), and 25 (Shexian County, Handan); (5) customers transported by Xingtai: 4 (Ninghai County, Tianjin), 15 (Zunhua City, Tangshan), 18 (Shunping County, Baoding), 19 (Renqiu County, Cangzhou), and 20 (Haixing County, Cangzhou).

The comparison of the optimization curves of the three algorithms is shown in Figure 4. As can be seen from
Figure 4, the FWA-artificial fish swarm algorithm has a faster optimization speed and a higher accuracy.

\section{Conclusion}

At first, this paper aims at minimizing the total cost and establishes a location model of low-carbon cold chain logistics distribution center, including cargo damage, refrigeration, carbon emission, and other factors, in combination with various factors affecting cold chain food. Secondly, the explosion operator, mutation operator, mapping rule, and selection strategy of the fireworks algorithm are introduced into the artificial fish swarm algorithm, and the FWA-artificial fish swarm algorithm is proposed. At the same time, the FWA-artificial fish swarm algorithm is applied to the location model of low-carbon cold chain logistics distribution center in the Beijing-Tianjin-Hebei metropolitan area. The simulation results show the effectiveness of the FWA-artificial fish swarm algorithm.

Optimizing the location of low-carbon cold chain logistics in the Beijing-Tianjin-Hebei metropolitan area not only makes the links between cities in the circle closer and promotes the integration of resources in the region but also is conducive to realizing the goal of low-carbon development; moreover, it is helpful to promote the economic development and economic integration process of the Beijing-Tianjin-Hebei region.

\section{Data Availability}

The data used for research and analysis are included within the article.

\section{Conflicts of Interest}

The authors declare that there are no conflicts of interest regarding the publication of this paper.

\section{Acknowledgments}

This work was supported by the Tianjin Natural Science Foundation (Grant no. 20JCYBJC00320).

\section{References}

[1] Z. Shuiyun: Thinking of "chain Breaking" behind cold chain logistics, 2019, http://www.zgsyb.com/news.html?aid=530826.

[2] Guangming, What is the potential of saving food, 2020, https://www.sohu.com/a/414789580_162758.

[3] Y. Marinakis, "An improved particle swarm optimization algorithm for the capacitated location routing problem and for the location routing problem with stochastic demands," Applied Soft Computing, vol. 37, pp. 680-701, 2015.

[4] J. Li, P. H. Fu, X. L. Li, J. H. Zhang, and D. L. Zhu, "Study on vehicle routing problem and tabu search algorithm under low-carbon environment," Chinese Journal of Management Science, vol. 23, no. 10, pp. 98-106, 2015.

[5] S. J. Sadjadi, A. Makui, E. Dehghani, and M. Pourmohammad, "Applying queuing approach for a stochastic location-inventory problem with two different mean inventory considerations," Applied Mathematical Modelling, vol. 40, no. 1, pp. 578-596, 2016. 
[6] Q. Z. Ma, J. Wang, and H. Q. Song, "Small-scaled low carbon multi-vehicle routing problem in urban area: an example from the regional picking-up network of Zhuhai express Company," Jonrnal of Industrial Engineering/Engineering Management, vol. 30, no. 4, pp. 153-159, 2016.

[7] J. A. Orjuela-Castro, L. A. Sanabria-Coronado, and A. M. Peralta-Lozano, "Coupling facility location models in the supply chain of perishable fruits," Research in Transportation Business \& Management, vol. 24, pp. 73-80, 2017.

[8] B. El-Sobky, Y. Abo-Elnaga, and L. Al-Naser, "An active-set trust-region algorithm for solving warehouse location problem," Journal of Taibah University For Science, vol. 11, no. 2, pp. 353-358, 2017.

[9] D. Simic, V. Ilin, V. Svircevic, and S. Simic, "A hybrid clustering and ranking method for best positioned logistics distribution centre in balkan peninsula," Logic Journal of IGPL, vol. 25, no. 6, pp. 991-1005, 2017.

[10] Y. Wang, Y. J. Ren, and M. Z. Xu, "Optimization of profit allocation based on multi-center joint distribution," Computer Integrated Manufacturing Systems, vol. 23, no. 7, pp. 1571-1580, 2017.

[11] K. Kang, J. Han, W. Pu, and Y. F. Ma, "Optimization research on cold chain distribution routes considering carbon emissions for fresh agricultural products," Computer Engineering and Applications, vol. 55, no. 2, pp. 259-265, 2019.

[12] F. T. Liang, J. K. Hu, and Y. F. Huang, "Electric vehicle routing problem under low carbon condition," Journal of Shanghai Maritime University, vol. 39, no. 2, pp. 34-40, 2018.

[13] O. N. Bilisik, U. R. Tuzkaya, H. Baracli, and M. Tanyas, "Fruits and vegetables market Hall location selection by using interval-valued trapezoidal fuzzy grey relational analysis an application for istanbul," International Journal of Industrial Engineering Theory Application and Practice, vol. 26, no. 5, pp. 719-736, 2019.

[14] Y. M. Zhang, Y. M. Li, and H. O. Liu, "Research on VRP optimization of multi-type vehicle cold-chain logistics with satisfaction constraint," Statistics \& Decisions, vol. 35, no. 4, pp. 176-181, 2019.

[15] L. J. Sheng, "Location of logistics distribution center based on quantum particle swarm optimization," Science Technology and Engineering, vol. 19, no. 11, pp. 183-187, 2019.

[16] J. H. Park, J. G. Oh, D. M. Kim, and G. T. Yeo, "A study on the establishment direction of smart distribution logistics center in the era of the fourth industrial revolution," Journal of Digital Convergence, vol. 17, no. 2, pp. 59-71, 2019.

[17] E. Setiawan, J. Liu, and A. French, "Resource location for relief distribution and victim evacuation after a sudden-onset disaster," IISE Transactions, vol. 51, no. 8, pp. 830-846, 2019.

[18] F. T. Zhou, G. H. Zhou, and J. Zhang, "Study on location of trans shipment points in urban distribution system based on subway network," Journal of the China Railway Society, vol. 41, no. 7, pp. 16-25, 2019.

[19] Y. H. Song, B. B. Su, F. Z. Huo, J. J. Ning, and D. H. Fang, "Research on rapid location selection of emergency materials distribution center considering dynamic demand," China Safety Science Journal, vol. 29, no. 8, pp. 172-177, 2019.

[20] S. Y. Li, B. Dan, and X. L. Ge, "Optimization model and algorithm of low carbon vehicle routing problem under multigraph time-varying network," Computer Integrated Manufacturing Systems, vol. 25, no. 11, pp. 2973-2982, 2019.

[21] J. Meng and J. H. Gao, "Location strategy of agricultural logistics distribution center based on improved moth optimization algorithm," Journal of Chinese Agricultural Mechanization, vol. 41, no. 4, pp. 207-214, 2020.
[22] B. Li and J. J. Dang, "Fresh agricultural cargoes locationrouting optimization with simultaneous pickup and delivery for multiple distribution centers," CAAI Transactions on Intelligent Systems, vol. 15, no. 1, pp. 50-58, 2020.

[23] Z. P. Li, Y. W. Zhao, and Y. W. Zhang, "Optimization model and algorithm of location-routing for joint distribution," Journal of Chongqing University, vol. 43, no. 1, pp. 28-43, 2020.

[24] D. S. Lin, Z. Y. Zhang, J. X. Wang, X. Liang, and Y. Q. Shi, "Low-carbon logistics distribution center location with uncertain demand," Control and Decision, vol. 35, no. 2, pp. 492-500, 2020.

[25] X. Q. Xu, Z. Yang, and L. Liu, "Spider monkey optimization algorithm for solving location problem of logistics distribution center," Computer Engineering and Applications, vol. 56, no. 1, pp. 150-157, 2020.

[26] W. Zhang, Y. Ma, H. D. Zhao et al., "Obstacle avoidance path planning of intelligent mobile based on improved fireworksant colony hybrid algorithm," Control and Decision, vol. 34, no. 2, pp. 335-343, 2019.

[27] C. Xiao, L. Y. Zhang, and T. Fei, "Research on bacteria foraging ant colony optimization algorithm for cold chain low carbon logistics distribution routing optimization," Mathematics in Practice and Theory, vol. 47, no. 21, pp. 98-107, 2017.

[28] T. Fei, L. Y. Zhang, Y. Bai, and L. Chen, "Improved artificial fish swarm algorithm based on DNA," Journal of Tianjin University, vol. 49, no. 6, pp. 581-588, 2016. 